\title{
Transiciones e hibridaciones en el profesionalismo docente: entre tecnologías de gobierno y gobernanza en espacios neoliberalizados
}

\author{
Luengo Navas, Julián \\ Universidad de Granada, Granada, España \\ jluengo@ugr.es \\ Molina-Pérez, Javier \\ Universidad de Granada, Granada, España \\ jmolinape@ugr.es
}

\section{Resumen}

El profesionalismo ha pasado por distintos periodos contingentes e históricos que descartan límites discretos, transiciones que arrastran características que perduran en el tiempo y otras que se van incorporando como consecuencia de las demandas producidas por los cambios políticos y sociales. La comunicación estudia la reestructuración de la profesionalidad docente como consecuencia de tecnologías políticas de gobierno, amparadas por procesos globales de neoliberalización y privatización, centrándose concretamente en lógicas resituadas de la Nueva Gestión Pública (NgP), y los nuevos modelos negociados de gobernanza en red que configuran la política pública. Se examina la transición desde la edad de oro de las burocracias profesionales y el profesionalismo ocupacional, caracterizado por la autonomía, discrecionalidad y certidumbre profesional hasta el profesionalismo organizacional, basado en la estandarización, la medición y la performatividad (Evetts, 2013). Posteriormente, en el contexto de la Nueva Gobernanza Pública (NGP), el profesorado continúa reprofesionalizándose hasta entenderse como un socio más de una red diversa conformada por agentes y actores interdependientes que intervienen en la gestación de la política (Hendrikx \& van Gestel, 2017). En ella, la autonomía y legitimidad profesional se definen dentro de los límites de la red, y su responsabilidad se dirime ante un grupo multifacético de partes interesadas (Brandsen \& Honingh, 2013).

\section{Abstract}

Professionalism has gone through different contingent and historical periods that discard discrete limits, transitions that drag characteristics that last over time and others that are incorporated as a result of the demands produced by political and social changes. The communication studies the restructuring of teaching professionalism as a consequence of government political technologies, protected by global neoliberalization and privatization processes, focusing specifically on the New Public Management (NPM) logics, and the new negotiated models of network governance that they shape public policy. It examines the transition from the golden age of professional bureaucracies and occupational professionalism, characterized by autonomy, discretion and professional certainty to organizational professionalism, based on standardization, measurement and performativity (Evetts, 2013). Subsequently, in the context of the New Public Governance (NPG), teachers continue re-professionalize themselves until they become a partner in a diverse network of agents and interdependent actors involved in the gestation of politics (Hendrikx \& van Gestel, 2017). In it, professional autonomy and legitimacy are defined within the limits of the network, and its responsibility is resolved before a multi-faceted group of interested parties (Brandsen \& Honingh, 2013).

Palabras clave: Neoliberalismo, Nueva Gestión Pública, Gobernanza, profesionalismo.

Keywords: Neoliberalism, New Public Management, Governance, professionalism.

\section{INTRODUCCIÓN}

La provisión de los servicios públicos debe ser analizada desde las diferentes lógicas que han articulado la promulgación e implementación de las políticas públicas. Si bien la posguerra estuvo caracte- 
rizada por una administración jerárquica, entendida como Administración Pública Tradicional (APT), la década de los ochenta y noventa del siglo anterior se caracterizó por el modelo denominado como Nueva Gestión Pública (NgP) (Pestoff, Brandsen \& Verschuere, 2012). Si la NgP consolidó en los servicios públicos una lógica empresarial para asegurar la eficiencia de la inversión presupuestaria, la gobernanza introduce la lógica comercial como elemento sustancial para la transformación que se produce en la provisión de éstos (Radnor \& Osborne 2013). La NgP, en primera instancia, alteró las formas de poder hegemónicas de la «edad de oro» de las burocracias profesionales. Posteriormente, el tránsito de gobierno hacia formas de gobernanza, caracterizadas por la negociación política entre distintos actores y agencias, ha propiciado un nuevo cambio en el rol de los profesionales de la educación. Estas cuestiones se analizarán a continuación prestando atención a los diferentes modelos de implementación de políticas públicas.

\section{ADMINISTRACIÓN PÚBLICA TRADICIONAL Y PROFESIONALISMO OCUPACIONAL}

Con el final de la Segunda Guerra Mundial y el auge de las políticas keynesianas en la era del Estado de Bienestar, se instaló la sólida creencia de que el Estado podía satisfacer todas las necesidades sociales y económicas de la población (Bryson, Crosby \& Bloomberg, 2014). Bajo estos planteamientos se fraguó el modelo de APT, que estableció límites claros entre la esfera de la política y de la administración mediante el ejercicio de un poder jerárquico y centralizado. La responsabilidad de la entrega del servicio público quedó en manos del conocimiento experto y la autonomía de los profesionales públicos (Osborne, 2010; Brandsen \& Honingh, 2013). Son, como apunta Le Bianic, formas de poder hegemónicas de la «edad de oro» de las burocracias profesionales (Bezes et al., 2012), basadas en lógicas que emanan de la autoridad colegial de los grupos profesionales que defienden los intereses propios de la profesión con el fin de establecer monopolios laborales (Evetts, 2013). Implica, además, un alto grado de autonomía y discrecionalidad, y el trabajo se rige por una sólida identidad profesional y un código ético ampliamente elaborado por los propios profesionales. A su vez, los fines laborales se alinearon con los objetivos públicos, la equidad, la justicia social y el bienestar de la ciudadanía. En el caso de la educación, las profesionales se enfocaron más en los ideales de la sociedad que en el cumplimiento de resultados, lo que se manifestaba en la autonomía, la inquietud intelectual y en la visión crítica de las tareas profesionales (Connell, 2009).

El profesionalismo, en el sentido clásico, es un fenómeno regulatorio (Noordegraaf, 2016), ya que los grupos ocupacionales están capacitados para controlar sus propias prácticas laborales, basándose en su conocimiento experto y en la experiencia. En general, esto se debe a que las asociaciones profesionales seleccionan, educan, socializan y supervisan a sus miembros profesionales, respaldadas por garantías estatales y vinculadas a instituciones académicas. No obstante, en el caso de las profesiones que se ocupan de la atención a las personas, como es la enseñanza, las bases de la profesionalidad se vinculan más con los entornos y las normas de la organización, porque su labor la han realizado en ámbitos institucionales (Noordegraaf, 2015). Sin embargo, con el inicio de la década de los ochenta del siglo pasado se introdujeron severos cambios en la gestión pública. Las reformas de la NgP se diseminaron con la potestad de redefinir la naturaleza y los objetivos de las organizaciones y de las profesionales del sector público.

\section{NUEVA GESTIÓN PÚBLICA Y PROFESIONALISMO ORGANIZACIONAL}

La NgP se desarrolla como un proyecto que obedece a la conjunción de factores políticos, relacionados con la hegemonía neoliberal y el auge de la Nueva Derecha; factores económicos, vinculados a la crisis fiscal y su consecuente limitación para la financiación de servicios públicos; y factores sociales, asociados a la demanda de la población de una mejora en la eficiencia y en la calidad de prestación del servicio público (Gunter, Grimaldi, Hall \& Serpieri, 2016). Como tal, prometió, y promete, mejorar los servicios públicos haciendo que las organizaciones estatales funcionen con patrones de desempeño análogos a los de la empresa privada (Diefenbach, 2009). Se pretendió desburocratizar las instituciones públicas mediante la implementación de "cánones capitalistas» que las hicieran más eficaces $\mathrm{y}$, ante todo, más eficientes, en un ejercicio sin precedentes de reconstrucción de la arquitectura del sector público (Jarl, Fredriksson \& Persson, 2011). La complejidad que exige definir de forma precisa qué es la NgP, lleva a no tratar de inmiscuirse en un debate taxonómico o epistemológico sobre sus teorías y prácticas. Más que eso, interesa saber que se trata de «una constelación de 
teorías gerenciales, marcos y herramientas, reglamentos y normas para la reforma del sector público producidas por redes transnacionales de expertos, policy-makers, organizaciones internacionales, think tanks, fundaciones y grupos de consultoría» (Verger \& Normand, 2015, p.614-615). Como tal, presenta unas características definitorias, pero las distintas tecnologías mediante las que opera precisan estudiar su recontextualización atendiendo a los fenómenos locales, los legados institucionales, políticos, culturales y legales (Gunter, Grimaldi, Hall \& Serpieri, 2016).

En educación, la $\mathrm{NgP}$ «incorpora políticas como la autonomía escolar, la rendición de cuentas, la publicación de los resultados en pruebas estandarizadas, la jerarquización, la profesionalización de los directores y la emulación de estilos gerenciales en la dirección escolar» (Parcerisa, 2016, p. 18). Con ello, la NgP consigue precisar cómo deben funcionar las organizaciones del sector público y las personas que trabajan en ellas. Los profesionales se convierten en empleados para la organización, y la confianza se define mediante la evaluación y la auditoría (Ferlie, Fitzgerald, McGivern, Dopson \& Bennett, 2013). Hendrikx y van Gestel (2017) caracterizan a los profesionales que trabajan en el contexto de la NgP como proveedores de servicios, pues su cometido laboral consiste en entregar un servicio público de calidad. En esta lógica, los procedimientos de trabajo están estandarizados porque el servicio educativo es diseñado y producido externamente por los intereses de los consumidores. La autonomía y la discrecionalidad de las profesionales son debilitadas por la cultura gerencial que controla las organizaciones (Freidson, 2001). La eficacia y la rendición de cuentas se establecen de forma objetiva (Osborne, 2010), y la satisfacción de los clientes se canaliza por medio de cuasi- mercados o incentivos docentes. Las profesionales se encuentran bajo la supervisión de la lógica organizacional, que define y valora sus competencias laborales (Evetts, 2012), al tiempo que establece las condiciones de trabajo que deben satisfacer las demandas educativas de las familias (Noordegraaf, 2011). Por ello, la base de legitimidad emana de la propia organización. La rigidez y la imposición de instrucciones, reglas y prácticas denuncian la desconfianza hacia la práctica experta del profesorado, que debe enfocar la enseñanza para satisfacer el interés particular de clientes aislados que buscan diferenciarse socialmente por medio de los resultados académicos. Keddie (2017) entiende que el «profesionalismo emprendedor» privilegia los sistemas de rendición de cuentas, el individualismo, la competencia y el cumplimiento de objetivos y resultados. Se trata de un "profesional performativo» que se interesa fundamentalmente por la superación de los estándares y el cumplimiento de los objetivos y resultados (Tseng, 2015). Un profesional que abraza el control, la regulación y la normalización; que asume programas académicos y prescriptivos sin tener en cuenta propósitos más amplios de la escolarización (West, 2010); que concibe la profesión como un oficio compuesto por un conjunto de habilidades y comportamientos que se aplican sin reflexión crítica; que utiliza una metodología basada en las evidencias de lo que funciona para obtener resultados (Wilkins, 2015). Cuestiones que indican un progresivo desvío y desatención de los objetivos públicos y del interés social, comunitario y democrático de la educación. No obstante, como advierten Klenk y Pavolini (2015), los análisis en el escenario actual deben ir más allá de la mercantilización o de la gestión, debido a la complejidad de amplias presiones políticas que reflejan deseos diversos y lógicas en pugna en la arena de la gestación de las políticas públicas en contextos sociales complejos.

\section{NUEVA GOBERNANZA PÚBLICA Y PROFESIONALISMO EN RED}

Las nuevas dinámicas de gobierno, consecuentes de los amplios procesos de privatización, así como la integración de recursos tecnológico-digitales y las nuevas formas de participación política, están abriendo paso a una era post-NgP (Lodge \& Gill, 2011). En ésta, la gobernabilidad prioriza la actividad de gestionar la influencia de nuevos actores que configuran la política pública, y que a veces la financian o la proveen, en detrimento de la gestión de la eficiencia de recursos que provenían del erario público (McLaughlin, Osborne \& Ferlie, 2002). En otras palabras, se ha cambiado el enfoque óptico y la gestión del gobierno, centrada en las organizaciones públicas, transita hacia modelos de gobernanza en los que nuevos y viejos actores, compran, venden y coproducen la política. En esta línea, Osborne (2010) considera que estas transiciones descartan límites discretos y artificiales, en tanto que los elementos de la NgP siguen copando gran parte de la agenda política. Por tanto, entiende que se produce una hibridación de prácticas y estructuras de gobierno que favorecen la participación de nuevos actores, al tiempo que se establecen nuevas dinámicas de mercado y cuasimercado por medio de redes interconectadas (Klijn \& Koppenjan, 2015). En realidad, se ha producido un "giro de gobierno» donde las redes de educación empresarial suplantan y sustituyen a actores públicos por su ineficiencia para la prestación del servicio. El desarrollo de una política horizontal selectiva ha supuesto una reestructuración y reconstrucción de la responsabilidad pública, en tanto que los distintos actores se presentan 
en redes policéntricas que difuminan los espacios entre lo público y lo privado (Oldham, 2017). Así, las nuevas modalidades de gobernanza se muestran bajo la influencia de los think tanks, las alianzas público-privadas, la gobernanza en redes heterárquicas, las nuevas configuraciones de la filantropía o el emprendimiento social. Las tendencias privatizadoras que el neoliberalismo extendió por medio de modelos mercadistas, están mutando y moviéndose dentro de amplias redes políticas compuestas por «empresarios de políticas» entre las que se encuentran filantrocapitalistas, think tanks, edu-empresas privadas, emprendedores y lobbistas. Es el proceso político el que está sometido a una privatización que conlleva que nuevas redes políticas, financiadas por actores y agencias privadas, intervengan en el discurso público y en la gestación de la política (Anderson \& Donchik, 2016).

Como advierte Noordegraf (2015, p. 132): «el profesionalismo actual es una cuestión de organización en contextos desorganizados». Hendrikx y van Gestel (2017) junto con Bradsen y Honing (2013) proponen que en los contextos profesionales en los que el conocimiento experto es disperso y está enfocado en los procesos y en las habilidades relacionales, el profesionalismo se define en comunidades inter-organizacionales dominantes. La autonomía está delimitada por la negociación en el seno de la red y la responsabilidad debe dar respuesta al grupo de actores interesados y las demandas de los clientes. La búsqueda de legitimidad e identidad profesional es una constante en este relato de gobernanza de las profesionales. Los profesores, como profesionales que operan en redes complejas (Rhodes, 2007), son conscientes de su falta de control en muchos ámbitos que legitiman su práctica diaria. Son desafiados constantemente en la tarea de «hacerse profesional» (Noordegraaf, 2007, p. 761), y en la delimitación de «territorios profesionales» (van den Berg \& van Reekum, 2011, p. 416), para definir su contenido profesional. Se observa que la lucha por el control institucional y el contenido profesional se realiza cada vez más fuera del poder de los propios profesionales, en redes de agencias y acuerdos en nuevas formas de gobernanza. Ello no ocurre en los contextos de la profesionalidad burocrática, en la que los «profesionales puros» aplican su conocimiento experto y técnico a casos concretos, siguiendo reglas rutinarias. En el contexto de la NGP, los profesionales se configuran como socios en la creación de políticas en el seno de la red, exigiéndose roles híbridos o múltiples para dar respuesta a demandas interprofesionales y multidisciplinares.

\section{DISCUSIÓN Y CONCLUSIONES: MÁS ALLÁ DE LA HIBRIDACIÓN}

Los sistemas de provisión pública nunca se han gobernado de una forma pura, sino que se han nutrido de la interacción de principios políticos, objetivos, valores o estructuras, que incorporan pautas culturales y sociales históricamente construidas (Kuhlmann \& Saks, 2008). Así, en los actuales contextos complejos y dinámicos, las profesionales forman roles híbridos (Noordrefraf, 2007; Colley \& Guéry, 2015) debido a la creciente permeabilidad entre los límites de sectores, organizaciones, prácticas o conocimiento, que implica la formación de nuevas perspectivas de trabajo. Sin embargo, en lugar de una inestable combinación de principios profesionales y organizacionales (Blomgren \& Waks, 2015), se observa que la organización se convierte en una parte normal del trabajo profesional que entrelaza ambos principios en las prácticas cotidianas. Se trata de incorporar la organización y la organización de roles y capacidades en el seno de la acción profesional. Así, organizar el profesionalismo se ocupa de establecer conexiones en los ámbitos laborales para abordar las tareas. Los profesionales organizados atienden a valores múltiples que les impele a establecer conexiones laborales diversas. Las emergentes condiciones sociales generan nuevas demandas en el ámbito profesional que exige acciones profesionales más organizadas, interrelacionadas, responsables y basadas en las partes interesadas, que las profesionales ya no ven como ajenas, sino normales. Esto no es profesionalismo mutado, sino reconfigurado (Noordegraaf, 2015). 


\section{REFERENCIAS BIBLIOGRÁFICAS}

Anderson, G. L., \& Donchik, L. M. (2016). Privatizing schooling and policy making: The American legislative exchange council and new political and discursive strategies of education governance. Educational Policy, 30(2), 322-364.

Bezes, P., Demazière, D., Le Bianic, T., Paradeise, C., Normand, R., Benamouzig, D., Pierru, F., \& Evetts, J. (2012). New public management and professionals in the public sector. What new patterns beyond opposition? Sociologie du travail, 54, e1-e52.

Blomgren, M., \& Waks, C. (2015). Coping with contradictions: hybrid professionals managing institutional complexity. Journal of Professions and Organization, 2(1), 78-102.

Brandsen, T., \& Honingh, M. (2013). Professionals and shifts in governance. International Journal of Public Administration, 36(12), 876-883.

Bryson, J. M., Crosby, B. C., \& Bloomberg, L. (2014). Public value governance: Moving beyond traditional public administration and the new public management. Public Administration Review, 74 (4), 445-456.

Colley, H., \& Guéry, F. (2015). Understanding new hybrid professions: Bourdieu, illusio and the case of public service interpreters. Cambridge journal of education, 45 (1), 113-131.

Connell, R. (2009). Good teachers on dangerous ground: Towards a new view of teacher quality and professionalism. Critical studies in education, 50(3), 213-229.

Diefenbach, T. (2009). New public management in public sector organizations: the dark sides of managerialistic 'enlightenment'. Public administration, 87(4), 892-909.

Evetts, J. (2012). Professionalism in Turbulent Times: Changes, Challenges and Opportunities. Propel International Conference. Nothingham: University of Nottingham.

Evetts, J. (2013). Professionalism: Value and ideology. Current Sociology, 61(5-6), 778-796.

Ferlie, E., Fitzgerald, L., McGivern, G., Dopson, S., \& Bennett, C. (2013). Making wicked problems governable?: The case of managed networks in health care. Oxford: Oxford University Press.

Freidson, E. (2001). Professionalism: the Third Logic: on the Practice of Knowledge. Chicago: University of Chicago Press.

Gunter, H. M., Grimaldi, E., Hall, D., \& Serpieri, R. (2016). New Public Management and the Reform of Education: European Lessons for Policy and Practice. London: Routledge.

Hendrikx, W., \& van Gestel, N. (2017). The emergence of hybrid professional roles: GPs and secondary school teachers in a context of public sector reform. Public Management Review, 19 (8), 1105-1123.

Jarl, M., Fredriksson, A., \& Persson, S. (2011). New public management in public education: A catalyst for the professionalization of Swedish school principals. Public Administration, 90 (2), 429-444.

Keddie, A. (2017). Supporting disadvantaged students in an English primary school: matters of entrepreneurial and traditional professionalism. Cambridge Journal of Education, 1-16. DOI: 10.1080/0305764X.2016.1270257.

Klenk, T., \& Pavolini, E. (eds.). (2015). Restructuring Welfare Governance: Marketisation, Managerialism and Welfare State Professionalism. Cheltenham, UK: Edward Elgar Publishing.

Klijn, E. H., \& Koppenjan, J. (2015). Governance networks in the public sector. London and New York: Routledge.

Kuhlmann, E., \& Saks, M. (Eds.). (2008). Rethinking professional governance: International directions in healthcare. Bristol: Policy Press.

Lodge, M., \& Gill, D. (2011). Toward a New Era of Administrative Reform? The Myth of Post-NPM in New Zealand. Governance: An International Journal of Policy, Administration, and Institutions, 24(1), 141-166.

McLaughlin, K., Osborne, S. P., \& Ferlie, E. (Eds.). (2002). The New Public Management: Current Trends and Future Prospects. London: Routledge.

Noordegraaf, M. (2007). From «pure» to «hybrid» professionalism: Present-day professionalism in ambiguous public domains. Administration \& Society, 39(6), 761-785.

Noordegraaf, M. (2011). Remaking professionals? How associations and professional education connect professionalism and organizations. Current Sociology, 59(4), 465-488.

Noordegraaf, M. (2015). Hybrid professionalism and beyond:(New) Forms of public professionalism in changing organizational and societal contexts. Journal of professions and organization, 2(2), 187-206.

Noordegraaf, M. (2016). Reconfiguring professional work: Changing forms of professionalism in public services. Administration \& Society, 48(7), 783-810.

Oldham, S. (2017). Enterprise Education: Critical Implications for New Zealand Curriculum Governance. New Zealand Journal of Educational Studies, 52(2), 331-346. 
Osborne, S. P. (2010). Introduction: The (New) Public Governance: a suitable case for treatment? En D. Osborne (Ed.), The new public governance? Emerging perspectives on the theory and practice of public governance (pp. 1-16). London: Routledge.

Parcerisa, L. (2016). Modernización conservadora y privatización en la educación: el caso de la LOMCE y la Nueva Gestión Pública. Revista Educación, Política y Sociedad, 2(1), 11-42.

Pestoff, V., Brandsen, T., \& Verschuere, B. (Eds.). (2012). New public governance, the third sector, and co-production. London: Routledge.

Radnor, Z., \& Osborne, S. P. (2013). Lean: a failed theory for public services?. Public Management Review, 15(2), 265-287

Rhodes, R. A. W. (2007). Understanding Gobernance: Ten Yeaars On. Organization Studies, 28, 1243-1264.

Tseng, C. Y. (2015). Changing headship, changing schools: how management discourse gives rise to the performative professionalism in England (1980s-2010s). Journal of Education Policy, 30(4), 483-499.

Van den Berg, M., \& van Reekum, R. (2011). Parent involvement as professionalization: professionals' struggle for power in Dutch urban deprived areas. Journal of Education Policy, 26(3), 415-430.

Verger, A., \& Normand, R. (2015). Nueva Gestión Pública y educación: elementos teóricos y conceptuales para el estudio de un modelo de reforma educativa global. Educação \& Sociedade, 36(132), 599-622.

West, M. (2010). School-to-school cooperation as a strategy for improving student outcomes in challenging contexts. School effectiveness and school improvement, 21(1), 93-112.

Wilkins, C. (2015). Education reform in England: quality and equity in the performative school. International Journal of Inclusive Education, 19(11), 1143-1160. 